\title{
Development status of adjustable grazing incidence optics for 0.5 arcsecond $X$-ray imaging
}

\author{
Paul B. Reid ${ }^{\mathrm{a}^{*}}$, Thomas L. Aldcroft ${ }^{\mathrm{a}}$, Ryan Allured ${ }^{\mathrm{a}}$, Vincenzo Cotroneo ${ }^{\mathrm{a}}$, \\ Raegan L. Johnson-Wilke ${ }^{\mathrm{b}}$, Vanessa Marquez ${ }^{\mathrm{a}}$, Stuart McMuldroch ${ }^{\mathrm{a}}$, Stephen L. O’Dell, \\ Brian D. Ramsey ${ }^{\mathrm{c}}$, Daniel A. Schwartz ${ }^{\mathrm{a}}$, Susan Trolier-McKinstry ${ }^{\mathrm{b}}$, Alexey Vikhlinin ${ }^{\mathrm{a}}$, \\ Rudeger H. T. Wilke ${ }^{\mathrm{b}}$, and Rui Zhao ${ }^{\mathrm{b}}$ \\ a Harvard-Smithsonian Center for Astrophysics, 60 Garden St. Cambridge, MA 02138 \\ b Pennsylvania State University, University Park, PA 16802 \\ c NASA Marshall Space Flight Center, Astrophysics Office (ZP12), Huntsville, AL 35812
}

\begin{abstract}
We describe progress in the development of adjustable grazing incidence X-ray optics for 0.5 arcsec resolution cosmic X-ray imaging. To date, no optics technology is available to blend high resolution imaging like the Chandra $X$-ray Observatory, with square meter collecting area. Our approach to achieve these goals simultaneously is to directly deposit thin film piezoelectric actuators on the back surface of thin, lightweight Wolter-I or WolterSchwarschild mirror segments. The actuators are used to correct mirror figure errors due to fabrication, mounting and alignment, using calibration and a one-time figure adjustment on the ground. If necessary, it will also be possible to correct for residual gravity release and thermal effects on-orbit.

In this paper we discuss our most recent results measuring influence functions of the piezoelectric actuators using a Shack-Hartmann wavefront sensor. We describe accelerated and real-time lifetime testing of the piezoelectric material, and we also discuss changes to, and recent results of, our simulations of mirror correction.
\end{abstract}

Keywords: x-ray optics, adjustable optics, active optics, piezoelectric, deformable mirrors, SMART-X, high resolution, lightweight optics

\section{INTRODUCTION}

Astronomers have pushed the frontiers of astrophysics to a time when the Universe was only a fraction of its present age. Beyond the current frontier, we can find the very first stars, black holes and galaxies forming in the Universe. To study these objects requires large telescopes with higher angular resolution and increased collecting area, at all wavelengths. At optical and infrared wavelengths, astronomers are building telescopes with mirrors up to $100 \mathrm{~m}$ in diameter equipped with active adaptive optics control to achieve resolution near or at the diffraction limit. At radio wavelengths, a "Square Kilometer Array" interferometer is being planned. For X-ray astronomy, half (0.5) arc-second resolution with a modest collecting area of a few hundred to a thousand square centimeters - the Chandra X-ray Observatory - opened a new frontier in astronomy. SMART- $\mathrm{X}^{1,2}$, a mission concept, would utilize adjustable optics to achieve Chandra-like resolution with 30 times the collecting area, enabling X-ray astronomy to reach the epoch of formation of the first galaxies and supermassive black holes, cosmological redshift $\mathrm{z} \sim 6-10$.

To meet the simultaneous challenges of large collecting area and high resolution, we are developing X-ray optics employing deformable bimorph mirror technology ${ }^{3}$ with thin lightweight mirrors. These mirrors are adjustable rather than active, as mirror figure error is corrected (adjusted) once or infrequently, as opposed to being changed constantly at several cycles/sec (active). In our approach, the mirror figure is corrected on the ground and once or infrequently on-orbit, if required. This enables the correction of figure errors due to mirror manufacturing, mounting induced deformations, modeled/actual gravity release, and modeled/actual on-orbit thermal effects. The

\footnotetext{
*Contact author (PBR) email: preid@cfa.harvard.edu _ phone: (617) 495-7233
} 
bimorph approach with lightweight mirrors means there is no heavy reaction structure, allowing the thin mirrors to be densely nested to achieve large collecting area at reasonable launch mass.

Our adjustable mirror approach employs a thin film of piezoelectric material deposited on the back of extremely thin mirrors ${ }^{4}$. The concept is shown in Figure 1. A uniform platinum ground electrode is first deposited on the back surface of the $0.4-\mathrm{mm}$ thick mirror, followed by $\sim 1.5-\mu \mathrm{m}$ layer of the piezoelectric material lead zirconate titanate (PZT). A pattern of discrete, independently addressable, platinum electrodes are lithographically deposited on top of the PZT layer (Fig. 1, left). Applying a voltage across the top and bottom platinum electrodes produces a local electric field normal to the mirror surface, resulting in a strain in the PZT material parallel to the mirror surface. This produces a local deformation (in the vicinity of the energized pixel, or cell), called an "influence function" (Fig. 1, center and right). The amplitude as a function of applied voltage and shape of the influence function for each piezo cell is calibrated. A least-squares fit or deconvolution is used to determine the individual voltage required for each piezo cell to minimize the mirror figure error thus optimizing optical performance. Thermally formed 0.4-mm thick Corning Eagle ${ }^{\mathrm{TM}}$ glass is used as the substrate for the adjustable optic.
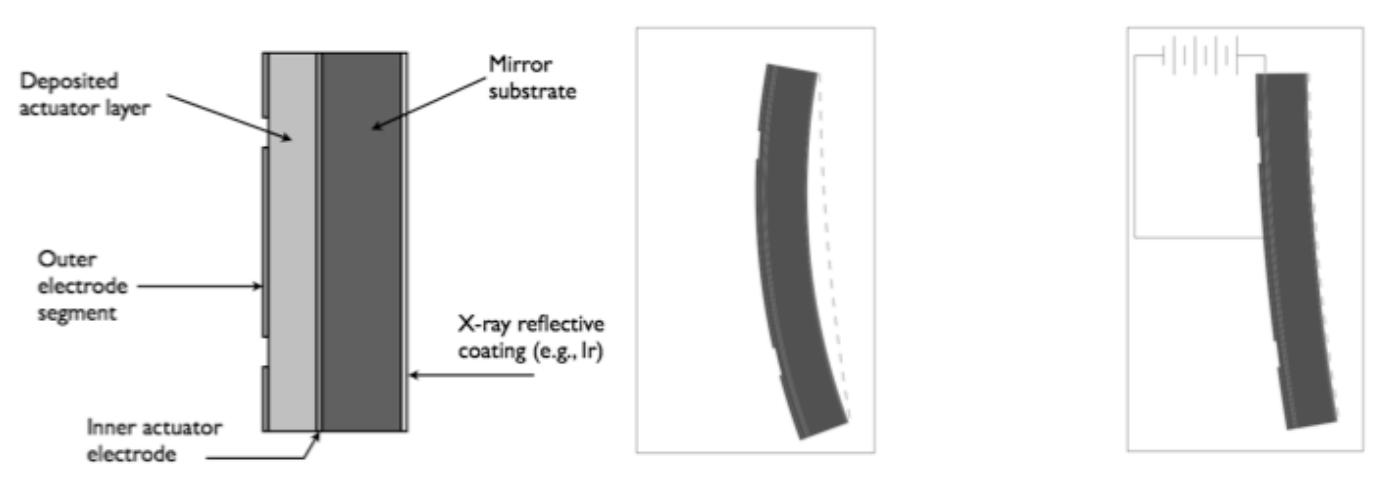

Figure 1. - Left: schematic of the bimorph active x-ray mirror (not to scale). Electrodes are divided into discrete, individually addressable cells. A figure error (middle) producing a deviation from the ideal (dotted line) is corrected by applying a DC voltage across the outer and inner electrodes (right). This produces a localized deformation that can be used to correct figure error.

The full telescope mirror assembly is built up from a number of these adjustable mirrors. Each individual mirror is a segment of a Wolter-I (or Wolter-Schwarzschild) primary or secondary, similar to the Constellation-X or International X-ray Observatory (IXO) slumped-glass concept ${ }^{5}$ : several mirror segments are co-aligned to form, e.g., a Wolter-I shell of a paraboloid of revolution and a hyperboloid of revolution. Many shells are nested together to produce the full collecting area.

\section{WHY ADJUSTABLE OPTICS?}

A valid question to ask is "Why adjustable X-ray optics?" Alternatively, why not develop some other technique which does not require electrical control of the mirrors on-orbit? Alternatives under development include (at least) non-contact thermal forming with ion implantation ${ }^{6}$, single-crystal silicon machined mirrors ${ }^{7}$, magnetostrictive adjustable mirrors ${ }^{8}$ that are adjusted only during fabrication, and differential deposition ${ }^{9}$. The answer to the question lies in the competing requirements of large effective area and high angular resolution. The SMART-X mission concept is aimed at Chandra-like imaging resolution with 30 times the collecting area $\left(2.3 \mathrm{~m}^{2}\right)$ at $1 \mathrm{keV}$. Similarly, the NASA Astrophysics Roadmap recommends, among others, an X-ray telescope mission called X-ray Surveyor with Chandra-like imaging and $>2 \mathrm{~m}^{2}$ effective area at $1 \mathrm{keV}^{10}$. Why do these requirements lead to a need for adjustable optics?

To achieve the necessary collecting area with a reasonable (or currently realistic) telescope mass, we require thin mirrors, as is being investigated in all the aforementioned concepts. Given a fixed maximum mass, maximum payload envelope, and similar focal lengths, the number of nested mirror shells will be inversely proportional to the mass per shell. The mass per shell is proportional to the mirror thickness. The telescope effective area (the collecting area), given these constraints, will be proportional to the number of shells. Thus the effective area is 
inversely proportional to the mirror thickness: double the mirror thickness and keep the same maximum mass, and the number of mirror shells and effective area are, to first order, halved.

Unfortunately, the stiffness of the mirrors scale basically as the thickness cubed. Halve the mirror thickness and the stiffness is reduced to $1 / 8^{\text {th }}$ of its previous value. Stray loads and moments on the mirrors, acting through the support points, introduce distortions in the mirror. The amplitude of the distortions is inversely proportional to the stiffness, so we can say that the amplitude of the distortions are inversely proportional to the thickness cubed. The point spread function (PSF) is, to first order, proportional to mirror figure slope error, which is proportional to the distortions. So now we have

$$
\begin{aligned}
& \text { Area } \propto 1 / \text { thickness } \\
& P S F \propto \text { thickness }^{3} .
\end{aligned}
$$

Decreasing the thickness allows us to increase the area, but makes the mirrors more susceptible to distortions that degrade (increase) the PSF. This identifies the trade space: Ignoring significantly larger launch vehicles in the near future, either we need to minimize the introduction of stray loads and moments due to mirror mounting and the onorbit thermal environment, or we need to have the capability to change - to correct - the figure after mounting and on-orbit, as required.

Of the alternative techniques to adjustable optics mentioned a few paragraphs ago, two - magnetostrictive and differential deposition - can be imagined to be employed post mirror mounting. But none of those alternative techniques are likely to be able to modify mirror performance on-orbit. Furthermore, we contend that adjustable optics technology can be melded with any of the four alternative approaches simply by adding the piezoelectric actuators to the back surface of the mirrors.

The error budget contributors for sub-arcsecond X-ray imaging are very small. Technologies that cannot account for mirror figure changes on-orbit or post-mounting have two difficult roads to follow: The mirrors must be made very accurately, and the mounting must eliminate very small stray loads and moments and be insensitive to on-orbit changes in the thermal environment. Adjustable optics technology only needs to follow the single difficult road of correcting mirror figure very accurately, and loosens the constraints on mounting and response to orbital environment. This discussion should not be construed as meaning adjustable optics are more likely to succeed at meeting the large-area, high-resolution, requirements than the other technologies. Rather, this presents the concept that adjustable optics provide an approach to simultaneously solve the parallel problems of making the mirrors "good enough" and making a system that is insensitive to small stray loads and changes in thermal environment.

\section{SUMMARY OF EARLIER PROGRESS}

In the past several years at the Smithsonian Astrophysical Observatory (SAO), we have modeled the sensitivity of the influence functions to the mirror mounting constraints, or boundary conditions ${ }^{11,12}$. We have determined that the influence functions are best localized and have higher bandwidth figure correction when the mirror mount overconstrains the mirror - i.e., the mirror is not supported kinematically. At the Materials Research Laboratory of the Pennsylvania State University (PSU), we developed the technology to sputter deposit PZT films 1.5- $\mu \mathrm{m}$ thick onto glass ${ }^{13,14,15}$ and found the resulting PZT films exhibit good piezo-electric properties that are consistent with meeting our system requirements for achieving figure correction to the 0.5-arcsec level. Measured performance of PZT actuators on both flat and cylindrical test mirrors is deterministic, that is, measured influence functions agree well with finite-element modeled (FEM, or FEA) influence functions, and are repeatable and stable ${ }^{16,17,18}$. We have demonstrated through simulation that the figure error of SMART-X sized mirror segments can be corrected to the 0.5 -arcsecond half power diameter (HPD) level ${ }^{19}$. These developments place thin film adjustable X-ray optics at or beyond NASA (and ESA) Technology Readiness Level (TRL) 3.

\section{RECENT TECHNOLOGY DEVELOPMENTS}

\subsection{Higher accuracy influence function measurement with a Shack-Hartmann wavefront sensor}

The accuracy with which influence functions are measured was improved by the incorporation of a ShackHartmann wavefront sensor. In earlier work, a scanning optical profilometer was used to measure influence functions ${ }^{16,17,18}$. Line scans through the center of the piezoelectric cell showed repeatability of $\sim 30 \mathrm{~nm}$ RMS. 
Surface scans, however, had repeatability of only 100-200 nm RMS, due to thermal variations occurring during the long time it took to acquire a surface map. While providing both the line scans and the surface scans demonstrated agreement between modeled and measured influence functions to better than metrology errors, the errors are too large either to demonstrate agreement with models, or to calibrate influence functions, at a level commensurate with half-arcsecond performance.

To improve our metrology accuracy, we incorporated a Shack-Hartmann wavefront sensor with a 128 pixel $\times$ 128 pixel lenslet array, along with beam-expander optics to produce a $125-\mathrm{mm}$ diameter plane wave for illuminating our test flat mirrors. (The beam diameter into the sensor is about $15 \mathrm{~mm}$.) Details of the Shack-Hartmann test and measurements are found in Allured et al. ${ }^{20}$. The Shack-Hartmann has the advantages of better absolute accuracy than the profilometer ( $<10 \mathrm{~nm}$ RMS versus $>20 \mathrm{~nm}$ RMS), better repeatability, and much faster measurement time. Wavefront sensor surface maps are obtained at an $\sim 10 \mathrm{~Hz}$ rate, compared to a full surface scan time with the profilometer of $\sim 30$ minutes to 1 hour. While spatial sampling is less with the Shack-Hartmann, simulation of pixelization effects on modeled influence functions reveal an insignificant impact; $2 \mathrm{~nm}$ peak-to-valley (PV).

Influence function measurements were made on multiple piezoelectric cells for several flat test samples. An example of such a measurement is shown in Figure 2, where the central piezoelectric cell was operated at $3 \mathrm{~V}$. Influence function measurements consist of averaging 10 seconds of data (100 images). A reference image is taken before energizing the piezoelectric actuator, and then the difference between voltage on and off yields the influence function. We note the peak displacement of $\sim 300 \mathrm{~nm}$ corresponds to an actuator "gain" of $0.1 \mu \mathrm{m} / \mathrm{V}$, consistent with earlier measurements made with the optical profilometer.

We also compared the measured influence functions with the finite-element modeled ones. The RMS difference over the full surface is $8.7 \mathrm{~nm}$. However, a significant part of the difference appears systematic rather than random. We investigated the repeatability of the wavefront sensor and mounted test mirror system and find a significant fraction of system repeatability is due to defocus as a result of the test mirror mount "breathing" and drifting, with

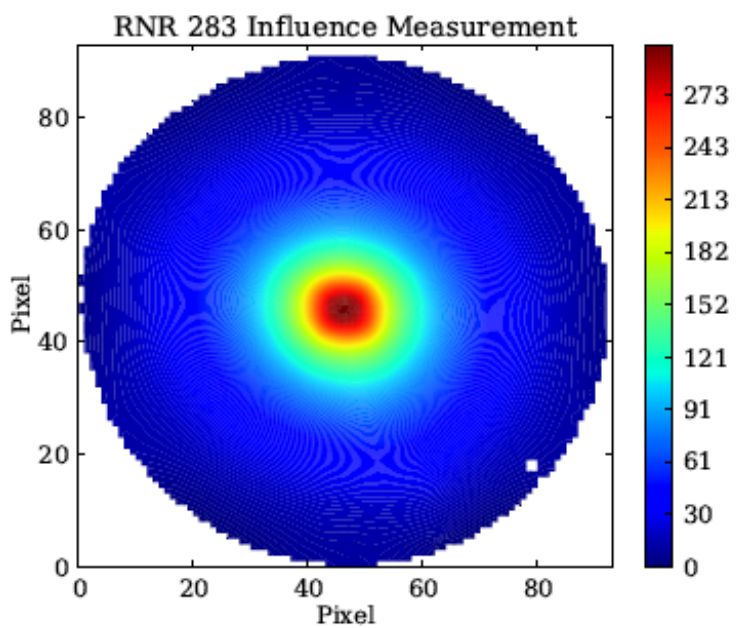

Figure 2. - Central influence function of a flat sample. The actuated cell was held at $3 \mathrm{~V}$, resulting in $\sim 300-\mathrm{nm}$ peak displacement. The color scale on the right is in units of $\mathrm{nm}$. additional systematic error appearing as hexafoil (6-fold azimuthal error), most likely due to the 6 point flexure mount (Figure 3). Repeatability can be as large as $6 \mathrm{~nm}$ RMS, including hexafoil and focus. Higher order repeatability (after focus and hexafoil) is 1-2 nm RMS. While it is unlikely that all the focus error is a result of system repeatability, we examined the significance of this term by removing its full contribution from the residual (this may represent close to a lower limit to the difference): This reduces the RMS difference between model and measurement to $3.6 \mathrm{~nm}$, shown in Figure 4. Axial registration of the wave-front sensor (WFS) $<2 \mathrm{~mm}$ accounts for $\sim<3 \mathrm{~nm}$ RMS difference. Lastly, we consider that a finite-element modeling error is typically considered to be in the $5 \%-10 \%$ range, or approximately 15 to $30 \mathrm{~nm}$, peak-to-valley for this influence function. Given these various error sources, we believe the measured influence functions agree with modeled influence functions (as we previously believed), but now to the better precision of between 8.7 and $3.5 \mathrm{~nm}$ RMS. In addition, we believe that this residual is well within the combination of system repeatability, metrology axial registration, and FE modeling error. We continue both to refine our measurement procedure to better account for system errors to attempt to further reduce the residual.

\subsection{Lifetime testing}

SMART-X, like most NASA flagships, will be planned as a 5-year-duration mission. But, as we have seen with Chandra (and XMM-Newton and Hubble), mission lifetimes can be significantly greater than originally planned. In addition, from the start of mirror fabrication to launch is also typically closer to 10 years. Thus we want as long a piezoelectric lifetime as possible. Two different types of lifetime testing are underway as part of the adjustable Xray optics programs: accelerated lifetime testing (ALT) at PSU, and real-time lifetime testing (RTLT) at SAO. 


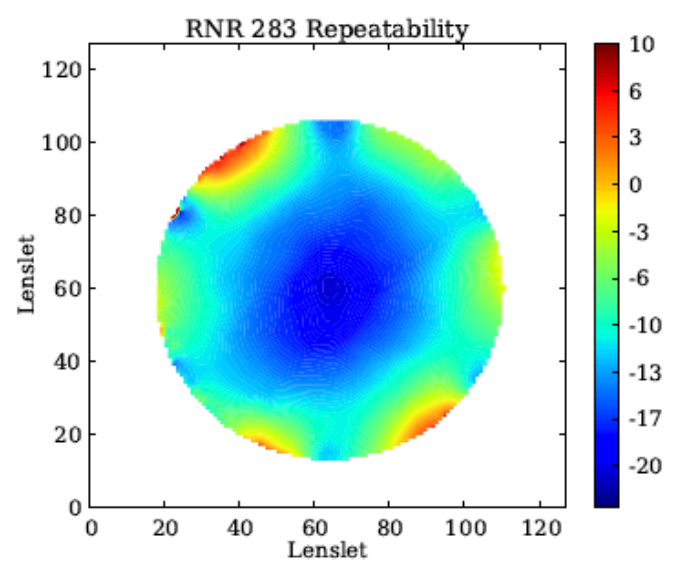

Figure 3. - Wavefront sensor repeatability, dominated by focus shift and hexafoil error. The vertical color scale is in units of $\mathrm{nm}$.

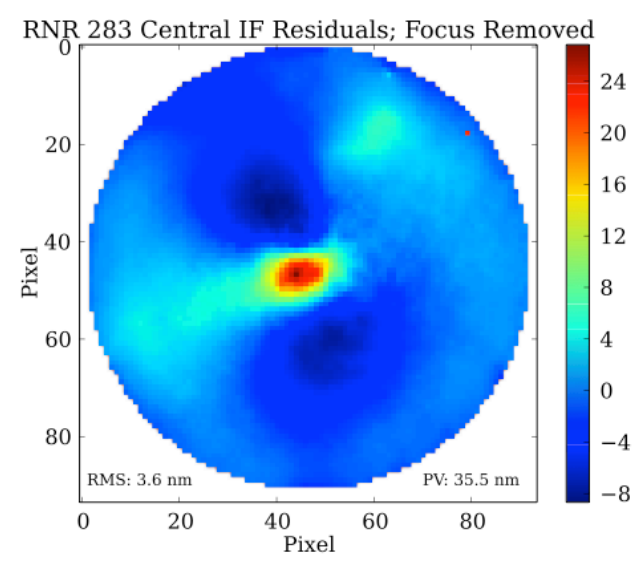

Figure 4. - Residual difference between measured and modeled influence functions, with focus removed. Vertical scale in units of $\mathrm{nm}$

Accelerated lifetime testing (ALT) is a standard technique in the microelectronics industry - including testing of PZT capacitors. Higher temperatures or increased electric fields (or voltages) are used to reduce operating lifetimes to reasonably testable durations. By testing over a range of temperatures and voltages, it is possible to infer the operating lifetime at "normal" operating conditions, via eq. (3).

$$
\frac{t_{1}}{t_{2}}=\left(\frac{E_{1}}{E_{2}}\right)^{-N} \cdot \exp \left[\frac{e_{a}}{k_{B}} \cdot\left(\frac{1}{T_{1}}-\frac{1}{T_{2}}\right)\right],
$$

where $t=$ lifetime, $E$ = electric field, $e_{a}$ is the activation energy, $k_{B}$ is the Boltzmann constant, $T$ is the temperature, and $N$ is the voltage acceleration factor. $N$ and $e_{a}$ are determined experimentally by varying the test control parameters of $E$ and $T$. In PSU's testing to date, the operating temperature was varied from $130^{\circ} \mathrm{C}$ to $240^{\circ} \mathrm{C}$, and the operating electric field ranged from $170 \mathrm{kV} / \mathrm{cm}$ to $300 \mathrm{kV} / \mathrm{cm}$. In contrast, normal operating conditions will be $21^{\circ} \mathrm{C}$ and $\left.<70 \mathrm{kV} / \mathrm{cm}\right)$. The accelerating conditions result in lifetimes of the test samples of $\leq \sim 100$ hours. However, using Weibull statistical analysis, the median time before failure for nominal temperature and maximum operating electric field is $\sim 1.6 \times 10^{10}$ hours ( 2 million years!). To increase testing statistics, though, smaller area piezoelectric cells are used. Additional testing is required to estimate the impact of increasing the cell area to our nominal plan (presently $0.5 \mathrm{~cm}^{2}$ ). In addition, once the area dependence is known, we need to correct for more typical operation at lower than maximum voltage, and using the Weibull parameters, determine the required lifetime for an allowable probability of failure during the mission of 1\%-2\% (see Section 4.3, below). These refinements to produce a lifetime estimate will be the subject of a future paper.

SAO is performing real-time lifetime testing to include effects of vacuum exposure, thermal cycling, and radiation exposure. We have built several vacuum chambers that each support multiple flat 100 -mm-diameter 0.4mm-thick Corning Eagle test mirrors with 1.5- $\mu$ m-thick PZT films. Deposition of the piezoelectric film and electrodes is done exactly the same as our current standard practice ${ }^{14,21}$. Each test mirror contains approximately 45 piezoelectric cells, each $1 \mathrm{~cm}^{2}$ in area. The test mirrors were poled (aligning the dipole moments of all the piezoelectric domains by applying a voltage to the cell), bonded to their 6-point mounts, re-poled, and then hysteresis curves were measured for each individual piezoelectric cell using the optical profilometer. The test mirrors and their mounts were installed in the test chambers, pumped down to $\sim 10^{-6}$ torr, and powered up. Based upon simulation results (Section 4.3), we determined that realistic operating voltages were in the range of 0 to $4 \mathrm{~V}$, and so operated the piezoelectric cells at either 2 or $4 \mathrm{~V}$, monitoring leakage current during testing. After $\sim 4$ weeks, the voltage was removed, the chamber was re-pressurized, and hysteresis curves were remeasured. After measurement, the pieces were returned to the test chamber, the chamber evacuated, and the test mirrors powered. 
We plan to test monthly, as least in the near term. Data is currently being reviewed and analyzed (at SAO and PSU). Figure 5 shows some preliminary results, where we plot relative gain (ratio of gain after 4 weeks of vacuum operation to gain before) on a piezoelectric cell-by-cell basis. (Gain is the ratio of peak displacement to maximum voltage during the hysteresis measurement.) Looking at the data we make several observations. First, we note (a) that there were no failures for the cells operated at $2 \mathrm{~V}$ and (b) that the $2-\mathrm{V}$ cells essentially are unchanged within errors: Their relative gain after/before remains 1 . Second, one 4-V cell (\#14) failed, although review of the leakage current shows this failure occurred at the start of the 4week test. Further, for the $4-\mathrm{V}$ cells, we see the ratio of after/before gains exceeds 1 , and is $\sim 1.7$. This indicates continued poling of the cells - i.e., they were not fully poled before testing. We expect subsequent measurements to show less, if any, increase in relative gain for the $4-\mathrm{V}$ cells. This result has also pointed out the need to pole the piezo cells more completely, something we are currently testing to optimize for stability and gain.

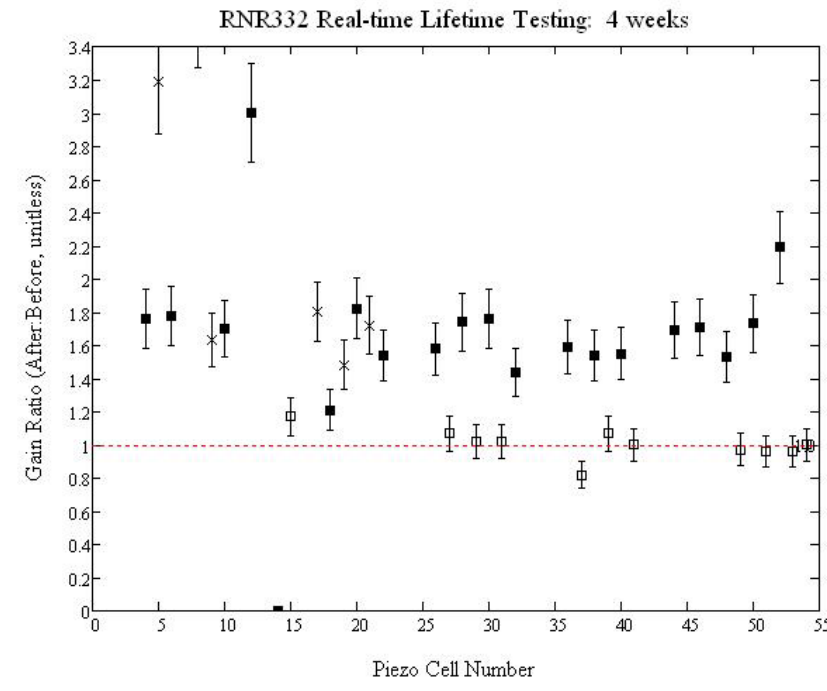

Figure 5. - Relative gain as a function of piezoelectric cell number for one of the two samples. Piezo cells run at $2 \mathrm{~V}$ (open squares) show relative gain equal to 1 , as expected. Piezo cells run at $4 \mathrm{~V}$ (closed squares) show higher gain after 4 weeks of vacuum operation due to continued poling of the piezoelectric cells.

\subsection{Improved piezoelectric cell voltage optimization and performance simulation}

We have developed the capability to optimize the set of piezoelectric voltages to minimize the point spread function of corrected mirrors and to predict the residual performance. Previously ${ }^{19}$, we used a linearized leastsquares optimization using residual error RMS amplitude as the merit function. Finite-element modeled influence functions were used, one for each adjuster, wherein look-up tables are used for each influence function. For improved simulation fidelity, influence function modeling includes a flexure design concept for mounting the mirrors. In the past year, we have transitioned to a "free-ware" bounded, constrained-least-squares optimizer ${ }^{22}$ using RMS axial slope error as the merit function. The bounds and constraints are used to require piezoelectric strains between 0 and 500 parts per million (basically, cell voltages between $0 \mathrm{~V}$ and $10 \mathrm{~V}$ ), again using look-up tables for modeled influence functions, one per cell. For simplicity, we set up our piezoelectric cell layout so there is "leftright” symmetry. Thus we only need to calculate half the influence functions. Once we have run the optimizer, we calculate the residual error map and compute performance either using RMS axial slope error, or via performing a full diffraction calculation using Kirchoff scalar scattering theory ${ }^{23}$. Depending upon the case under analysis, simulations are run for either SMART-X size mirror segments (20-cm long axially, and 20-40-cm wide azimuthally), or mirror segments sized for TRL 4 X-ray testing planned for 2015 (10-cm axially by 10-cm azimuthally, with the size dictated by the available PZT sputtering chamber). Obviously, different influence functions are used for the different mirror sizes.

As examples of the use of the simulation tools, we compared the figure correction possible with two different sized piezoelectric cells, as well as with different width gaps between the cells. In all three cases, we used as exemplar data interferometric measurements of the figure of a Con-X hyperboloid (secondary) mirror segment produced by W. Zhang at NASA GSFC (ca. 2008). The mirror was measured while bonded (mounted) in the ConX/IXO Optical Assembly Pathfinder (OAP) 2 mount $^{24}$. Data was obtained for an $\sim 20 \times 20 \mathrm{~cm}^{2}$ mirror and then "stretched" in the azimuthal direction to account for the larger span of a SMART-X mirror. The uncorrected performance for a mirror segment pair where each mirror segment's figure error is statistically identical to the exemplar data, but uncorrelated to one another, was 10-arcsec HPD and 14-arcsec RMS diameter (RMSD) at 1 keV with a graze angle of 0.025 rad (1.43 deg), corresponding to a 1-m mirror cylindrical radius and a 10-m focal length. Using piezoelectric cells 10 -mm axial $\times 20$-mm azimuthal, with a 1-mm gap between cells, yielded an RMSD residual of 1.2 arcsec at $1 \mathrm{keV}$. Changing to smaller piezoelectric cells -5 -mm axial $\times 10$-mm azimuthal - with a 
0.2-mm gap, resulted in an improvement in the corrected residual to 0.4 arcsec RMSD at $1 \mathrm{keV}$. Figure 6 shows a comparison of the solutions for the two cases. The top row shows contour plots of the solution for the $10 \mathrm{~mm} \times 20$ $\mathrm{mm}$ cells (and exemplar data), and the bottom row shows the solutions for the $5 \mathrm{~mm} \times 10 \mathrm{~mm}$ piezoelectric cells with $0.2-\mathrm{mm}$ gap between cells. On the left are the resulting piezo cell coefficients, representing the piezo strain in units of $100 \mathrm{ppm}$. On the right are post-correction axial slope errors. We point out several things in the plots. (1) The range of PZT strains that optimize performance is relatively low - from a few 10 's of ppm to $<200 \mathrm{ppm}$, ignoring the edges. This implies most of the piezo cells will be operating at well below their maximum $10 \mathrm{~V}$, which approximately corresponds to $500 \mathrm{ppm}$ strain. (2) We note the residual ripple in the slope plots on the right. The impact of this ripple is included in the performance prediction. While not apparent from the top and bottom plots, the residual ripple is significantly lower for the $5 \times 10 \mathrm{~mm}^{2}$ case. (3) The vertical striping in all our plots is due to the mount constraints, which were taken as 5 attachment points at each of the forward and aft ends (top and bottom) of the mirrors. As part of our future simulation activities, we will explore ways to try to minimize this effect. As a result of this simulation, we are developing our X-ray test optics with the smaller sized piezoelectric cells.
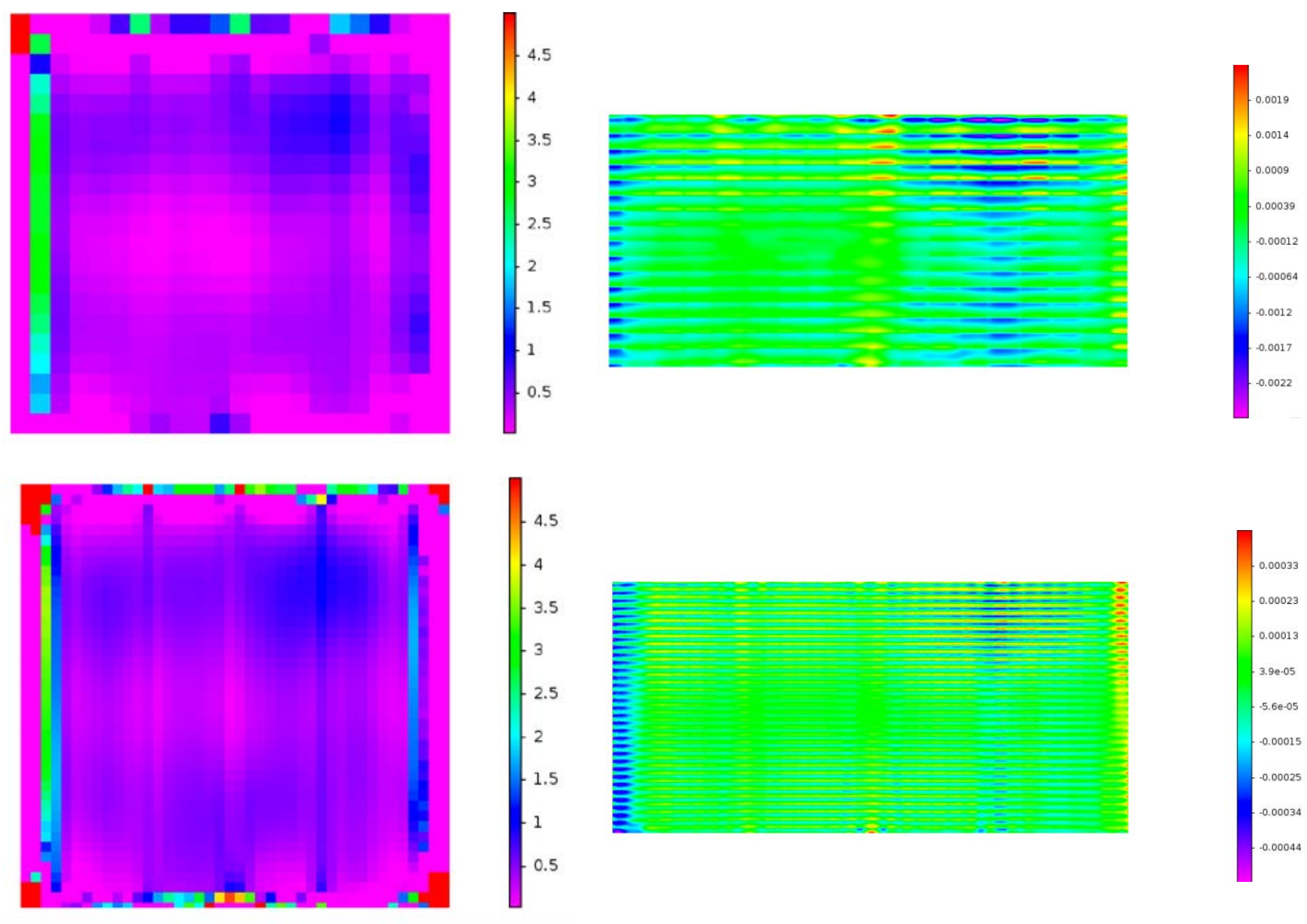

Figure 6. - Outputs from bounded constrained-least-squares optimizer for $10 \mathrm{~mm} \times 20 \mathrm{~mm}$ piezo cells (top row) and for $5 \mathrm{~mm} \times$ $10 \mathrm{~mm}$ piezo cells (bottom row). In all panels, the axial direction is vertical on the page, and the azimuthal direction is horizontal. The left side contour plots are the piezoelectric cell coefficients, which range from 0.0 to $5.0 \times 100 \mathrm{ppm}$ strain. The right side contour plots are the residual (after correction) surface axial slope error. 
The simulation tools can be used to determine how well cell displacement must be controlled. Using the cell voltage solution for the $5 \times 10 \mathrm{~mm}^{2}$ cells with 0.2 -mm gap design, the solution was randomly perturbed by a fraction of applied displacement. Results, shown in Figure 7, demonstrate that controlling the combination of cell drive voltage and piezoelectric coefficient to an equivalent of 1 ppm strain in the piezoelectric material is sufficient to maintain 0.5-arcsec RMSD imaging. Given that the nominal solution requires PZT strains of basically 30 to $150 \mathrm{ppm}$, this translates to an $\sim 0.7 \%$ requirement on control of the drive voltage or piezoelectric coefficient.

Finally, another application of the simulation tools is to evaluate probability of failures required to maintain performance during mission life (which would also include manufacturing yield). Again a Monte Carlo analysis is run, where $N$ randomly distributed piezoelectric cells are turned off — shorted to ground and a new optimized solution is obtained. The value of $N$ for which performance degrades above acceptable limits tells us the required maximum failure rate. Earlier analysis $^{19}$ showed an allowable combined manufacturing yield plus failure rate of $\sim 1 \%$, if one determines that a particular piezoelectric cell has failed and redoes the optimization for the remaining operational cells.

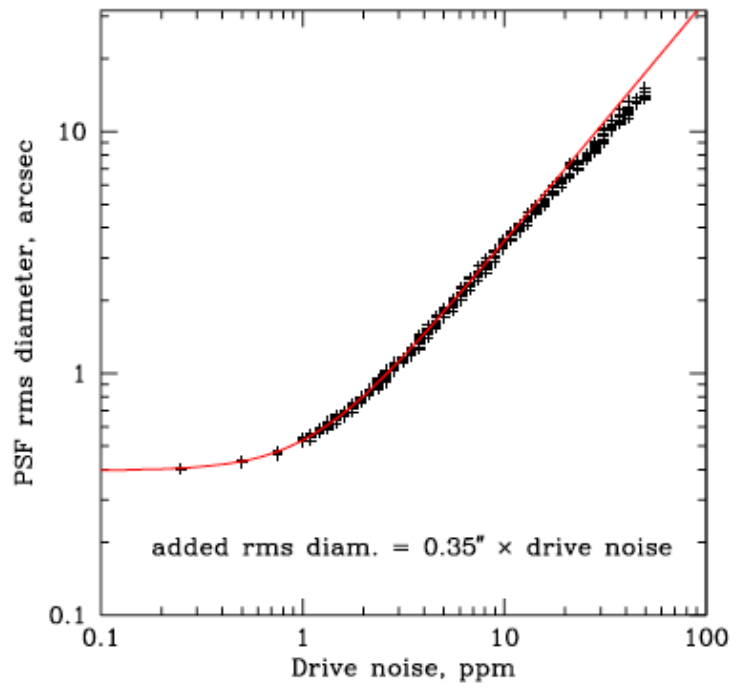

Figure 7. - Piezoelectric cell random drive noise impact on imaging performance. Drive noise is expressed as an equivalent strain in the PZT material. Strains in the nominal solution mostly range from 100 to $200 \mathrm{ppm}$.

\section{FUTURE PLANS/DEVELOPMENTS}

We are continuing to develop adjustable grazing incidence X-ray optics. Our current focus is to prepare a pair of aligned, mounted, corrected Wolter-I mirror segments for X-ray testing at the NASA MSFC Stray-Light Facility (SLF) X-ray beamline. We seek to use the results of X-ray testing, planned for late 2015, to demonstrate the end-toend feasibility of our approach. To that end, we are (in parallel) developing the capability to align mirror segment pairs to 0.35 arcsec RMSD. We will also be developing the necessary piezoelectric adjuster drive electronics to computer control all the adjuster cells simultaneously.

\section{SUMMARY}

We are continuing the development of adjustable grazing incidence X-ray optics. Adjustable X-ray optics represent a technical approach to large-area sub-arcsec imaging that eases constraints on the mirror mounting system and thermal environment. We have improved our metrology capability and newly measured influence functions show excellent agreement with modeled influence functions, at a more precise level than we demonstrated in the past and also consistent within our metrology and modeling expectations. Both accelerated and real-time lifetime testing of the piezoelectric material is underway, with promising early results. Our computer simulation tools have been improved via the incorporation of a bounded, constrained least-squares optimizer using mirror RMS slope error as the merit function for determining voltages for the piezoelectric cells that minimizes the RMS diameter of the image point spread function. The simulation tools were used to determine that mirror performance can be better optimized with smaller piezoelectric cells than we originally envisioned, and that minimizing the gap between cells also enables improved performance. Via simulation we demonstrated that mirror performance can be corrected from 10 arcsec HPD (14 arcsec RMSD) to < 0.1 arcsec HPD (0.4 arcsec RMSD), consistent with SMART-X requirements.

\section{ACKNOWLEDGMENTS}

This work was supported by NASA Contract NNX13AD46G and by internal funding from the Smithsonian Astrophysical Observatory. 


\section{REFERENCES}

[1] Vikhlinin, A., et al., "SMART-X, Square Meter Arc Second Resolution X-ray Telescope,” white paper submitted in response to NASA RFI NNH11ZDA018L, http://pcos.gsfc.nasa.gov/studies/x-ray/x-ray-mission-rfis.php (2011).

[2] Vikhlinin, A., et al., "SMART-X: a large area high resolution X-ray observatory for the 2020s," SPIE Proc. 8443, paper 42 (2012).

[3] Wilson, R.N., et al., "Active Optics I. A system for optimizing the optical quality and reducing the costs of large telescopes,” J. Mod. Opt. 34, 485 (1987).

[4] Reid, P., et al., "Development of adjustable grazing incidence optics for Generation-X,” SPIE Proc. 7011, 70110V (2008).

[5] Petre, R., et al., "Constellation-X spectroscopy X-ray telescope (SXT),” SPIE Proc. 4851, 433 (2003).

[6] Chalifoux, Brandon, et al., " Shaping of thin glass X-ray telescope mirrors using air bearing slumping and ion implantation,” SPIE Proc. 9144, 91444D (2014).

[7] Riveros, Raul E., et al., “ Fabrication of single crystal silicon mirror substrates for X-ray astronomical missions,” SPIE Proc. 9144, 914445 (2014).

[8] Ulmer, Melville P., et al., " Update to an application using magnetic smart materials to modify the shape of an X-ray telescope mirror,” SPIE Proc. 8861, 88611R (2013).

[9] Atkins, C., et al., “ X-ray optic developments at NASA’s MSFC,” SPIE Proc. 8777, 87770W (2013).

[10] C. Kouveliotou, et al., "Enduring Quest, Daring Visions: NASA astrophysics in the next three decades," NASA, 2013.

[11] Reid, Paul B. et al., "Generation-X mirror technology development plan and the development of adjustable x-ray optics," SPIE Proc. 7437, 74371F (2009).

[12] Davis, William N., et al., "Finite element analyses of thin film active grazing incidence x-ray optics," SPIE Proc. 7803, 78030P (2010).

[13] Wilke, Rudeger H.T., et al., "PZT piezoelectric films on glass for Gen-X imaging," SPIE Proc. 7803, 780300 (2010).

[14] Wilke, Rudeger H.T., et al., "Sputter deposition of PZT piezoelectric films on thin glass substrates for adjustable X-ray optics,” Appl. Opt. 52, 3412 (2013).

[15] Johnson-Wilke, R.L., et al., "Improving yield of PZT piezoelectric devices on glass substrates,” SPIE Proc. 8503, 85030A (2012).

[16] Cotroneo, V., et al., "Adjustable grazing incidence x-ray optics: measurement of actuator influence functions and comparison with modeling," SPIE Proc. 8147, 81471R (2011).

[17] Reid, P.B., et al., "Technology development of adjustable grazing incidence X-ray optics for sub-arc second imaging,” SPIE Proc. 8443, 84430T (2012).

[18] Reid, P.B., et al., "Development status of adjustable grazing incidence optics for 0.5 arc second X-ray imaging,” SPIE Proc. 8861, 88611Q (2013).

[19] Aldcroft, Thomas L., et al., "Simulating correction of adjustable optics for an X-ray telescope,” SPIE Proc. 8503, 85030F (2012).

[20] Allured, R., et al., "Measuring the performance of adjustable X-ray optics with wavefront sensing," SPIE Proc. 9144, 91441D (2014).

[21] Johnson-Wilke, R., et al., SPIE Proc. 9208, these Proceedings (2014).

[22] Gould, N.I.M., et al., "Galahad, a library of thread-safe Fortran 90 packages for large-scale nonlinear optimization,” ACM Trans. Math. Software 29 (4), 353 (2004).

[23] Beckmann, P., and Spizzichino, A., The Scattering of Electromagnetic Waves from Rough Surfaces (Artech House, Norwood MA), p24 (1987).

[24] Freeman, M.D., et al., "Progress on the active alignment system for the IXO mirrors," SPIE Proc. 7437, 74371E (2009). 